\title{
Das politische System des Südsudans
}

\author{
Von Ingo Henneberg, Augsburg*
}

\begin{abstract}
The Republic of South Sudan - after the secession from the former Sudan in July 2011 the youngest state in the world-faces a multitude of conflicts threatening the future of the country. For future research on South Sudan, a fundamental understanding of the new established system of government is highly essential. This article develops a first scientific overview of the new political system created by the Transitional Constitution of the Republic of South Sudan (2011) and analysis the constitutional settings (incl. the three branches of government, checks and balances and the legislative process), the developing party system as well as the media landscape, regional politics and (as possible) civil participation. This analysis classifies the new state within debates of scholars of political science and offers a simplifying diagram for a fast understanding of the new system.
\end{abstract}

$$
* * *
$$

\section{A. Einleitung}

Der Südsudan ist der jüngste Staat der Welt und steht vor einer Vielzahl von Herausforderungen. Um sich diesen stellen zu können braucht er ein politisches System, welches Legitimität erzeugen und Probleme praktisch lösen kann. Staaten und internationale Organisationen, die vielfach Hilfe beim Staatsaufbau zugesagt haben, benötigen profunde Kenntnisse des neuen Systems um effektiv kooperieren zu können und auch für weitergehende Forschung sind solche grundlegenden Informationen unerlässlich. Eine detaillierte Darstellung und wissenschaftlichen Kategorisierung des politischen Systems des Südsudan (Ganub as-Sudan) ist bisher weder in deutscher noch in englischer Sprache verfügbar. ${ }^{1}$ Auch der Blick auf die operative Politik hilft hierbei nicht weiter, die Informationen des Auswärtigen Amts (7 Zei-

* Ingo Henneberg, B.A. (ingo.henneberg@freenet.de) schließt zur Zeit sein Masterstudium der Sozialwissenschaftlichen Konfliktforschung an der Universität Augsburg ab. Thema der Masterarbeit: „Unmaking Wars. The Implementation of Power-Sharing Agreements in Angola, Sierra Leone, Liberia and Sudan" in Anlehnung an ein DFG-Forschungsprojekt am German Institute of Global and Area Studies (GIGA) in Hamburg. Dieser Text entstand anknüpfend an ein gemeinsames Forschungsprojekt mit Maik Boldau und Friedrich Plank an der Universität Augsburg. Beiden sei an dieser Stelle sehr herzlich gedankt.

1 Stand 2012. Einige hilfreiche Informationen, aber keine Beschreibung des Gesamtsystems liefern Andreas Auer / Corsin Bisaz / Fernando Mendez / Daniel Thürer, The Transitional Constitution of the Republic of South Sudan 2011. An Expert View from the Outside, Zentrum für Demokratie Aarau, 31.10.2011, www.uzh.ch/news/articles/2012/der-ruf-nach-demokratie-wird-immer-lauter/SouthSuda n2011.pdf (10.4.2013). Der Text wird mit Oktober 2011 datiert, wurde aber erst 2012 veröffentlicht. 
len $)^{2}$ und des US State Departments (13 Zeilen $)^{3}$ bleiben mehr als oberflächlich. Diese Analyse versucht diese Forschungslücke so gut dies die schwierige Quellenlage zulässt zu schließen ${ }^{4}$ und das etablierte System auf Basis der vorhandenen Informationen bestmöglich zu beschreiben. Dieser stark deskriptive Ansatz versteht sich dabei als Grundlagenforschung und Ausgangspunkt für weitere politikwissenschaftliche, rechtswissenschaftliche wie interdisziplinärer Analysen.

Für die Beschreibung der Systemgrundlagen wurde hauptsächlich auf die Verfassung Bezug genommen, bei der Parteilandschaft auf die Eigenaussagen der Parteien. Sehr schwierig gestaltet sich die Quellenlage im Bereich der Medienlandschaft sowie der Politik auf Ebene der Gliedstaaten und Regionen. Daneben schränkt die Neuartigkeit des Systems die Analysemöglichkeiten ein. Einschätzungen zur politischen Kultur können daher nur rudimentär gegeben werden, da sich dieser in einem neuen politischen System erst entwickeln muss, ebenso lassen sich zum jetzigen Zeitpunkt kaum Aussagen über die Verfassungswirklichkeit treffen. Zentral ist daher die Beschreibung des Verfassungssystems und seiner Ausprägungen. ${ }^{5}$ Hierbei wird in Abschnitt B zunächst die Verfassungsentwicklung und -Prinzipien vorgestellt, in Abschnitt C, D und E auf das Parlament, die Regierung und den Gesetzgebungsprozess eingegangen, die Abschnitte F, G und H gehen auf Parteien, Verbände und Medienlandschaft ein, nach der Beschreibung des Rechtssystems sowie der Regionalpolitik (Abschnitte I \& J) erfolgt abschließend ein Blick auf die internationalen Beziehungen (K) sowie ein Ausblick und die grafische Darstellung des Gesamtsystems.

Alle Rechtsquellen in Klammern beziehen sich jeweils auf die südsudanesische Verfassung von 2011.

2 Vgl. Auswärtiges Amt, Länderinformation Südsudan, 2012, www.auswaertiges-amt.de/DE/Aussenp olitik/Laender/Laenderinfos/01-Nodes_Uebersichtsseiten/Suedsudan node.html (10.4.2013).

3 Vgl. US-State Department, Background Note. South Sudan, 2012, www.state.gov/r/pa/ei/bgn/17171 8.htm\#gov (10.4.2013).

4 Zwar liegt ein Bericht der International Crisis Group (ICG) aus dem Frühjahr 2011 vor (vgl. ICG, Politics and Transition in the New South Sudan, Crisis Group Africa Report No. 172, 4.4.2011, www.crisisgroup.org/ /media/Files/africa/horn-of-africa/sudan/172\%20-\%20Politics\%20and $\% 20 \mathrm{Tr}$ ansition $\% 20$ in $\% 20$ the $\% 20 \mathrm{New} \% 20$ South $\% 20$ Sudan.ashx (10.4.2013)), jedoch beschreibt dieser weder vollständig das politischen System des Südsudans, noch nimmt er aufgrund seines Publikationsdatums aktuelle Entwicklungen nach der Unabhängigkeit mit auf.

5 Dies erfolgt in grober Anlehnung an das von Wolfgang Ismayr seit 1997 in mehreren Auflagen erschienen Standardwerk im Bereich der vergleichenden System- und Regierungslehre, nämlich Wolfgang Ismayr (Hrsg.), Die politischen Systeme Westeuropas, Wiesbaden 2009. 


\section{B. Verfassungsentwicklung und Verfassungsprinzipien}

Das politische System des 2011 neugegründeten Staates Südsudan basiert auf der „Transitional Constitution of the Republic of South Sudan" von 2011. ${ }^{6}$ Diese wurde auf Grundlage der „Interim Constitution of Southern Sudan"7 von 2005 ausgearbeitet und trat mit Unterschrift des Präsidenten der Übergangsregierung des „Südlichen Sudans“ (Verfassung von 2005) am 9. Juli 2011 in Kraft. Die Verfassung von 2011 stellt dabei eine in den wesentlichen Verfassungsbestandteilen nur aktualisierte Fassung der Verfassung von 2005 da, die freilich um einige wichtige Faktoren, die aus der Umstand einer eigenen Staatlichkeit folgen, erweitert wurde. ${ }^{8}$

Grundprinzipien der Verfassung sind die Volkssouveränität (Art. 2), die Gewaltenteilung und die Menschenrechte die in einem sehr umfangreichen Grundrechtekatalog (Bill of Rights) (Art. 9-34) niedergelegt sind. Staat und Religion sind getrennt (Art. 8).

In Art. 86 „Provisional Orders“, Provisorische Verordnungen des Präsidenten, findet sich in Abs. 5 eine Aufstellung des Verfassungskerns (vergleichbar Art. 20 GG) in den nicht eingegriffen werden darf, hierzu zählen: „Bill of Rights, the decentralized system of government, general elections, annual allocation of resources and financial revenue, penal legislation or alteration of administrative boundaries of the states. " Ähnliche Bestimmungen finden sich in Art. 190, in der besondere Grundrechte auch in Zeiten eines Ausnahmezustands notstandsfest gemacht werden. Genannt werden dort ,right to life, prohibition against slavery, prohibition against torture, the right of non-discrimination on the basis of race, sex, religious creed, the right to litigation or the right to fair trial", dies deckt sich größtenteils mit den völkerrechtlichen Verpflichtungen des Internationalen Paktes über bürgerliche und politische Rechte (Art. 2 (1)+(2) IPBPR), denen der Südsudan allerdings noch nicht zugestimmt hat. ${ }^{9}$

Die Grundrechte werden sowohl vom Obersten Gerichtshof, als auch von einer unabhängigen Menschenrechtskommission geschützt (Art. 9 (4) + Art. 10). Internationale Menschenrechtsverträge haben Verfassungsrang, sobald sie ratifiziert wurden (Art. 9 (3)).

6 Vgl. Transitional Constitution of the Republic of South Sudan, 2011, www.gurtong.net/LinkClick.as px?fileticket=SHBZGArJ8Ks\%3d\&tabid=106 (10.4.2013).

7 Vgl. Interim Constitution of Southern Sudan, 2005, www.chr.up.ac.za/undp/domestic/docs/c_Southe rnSudan.pdf (10.4.2013). Beide Verfassungen sind nicht auf offiziellen staatlichen Webseiten zu finden.

8 Der Autor erstellte gemeinsam mit Maik Boldau und Friedrich Plank eine umfangreiche Synopse der Verfassungen von 2005 und 2011, welche auf Anfrage herausgeben wird.

9 UN-Treaties, Statues of Treaties. International Covenant on Civil and Political Rights, 2012, http://tr eaties.un.org/Pages $/$ ViewDetails.aspx? src=TREATY\&mtdsg_no=IV-4\&chapter $=4 \&$ lang=en (10.4.2013). 
Die Republik Südsudan ist nach den etablierten Systematisierungen politischer Systeme sowohl nach Winfried Steffani ${ }^{10}$ als auch nach Arend Lijpart ${ }^{11}$ ein Präsidentielles Regierungssystem. Mangels der Position eines Premierministers entfällt die mögliche Einteilung als semi-präsidentielles System (nach dem frz. Politologen Maurice Duverger). ${ }^{12}$ Wie in präsidentiellen Systemen üblich herrscht im Südsudan eine recht starke Gewaltenteilung (z.B. Verbot für Minister ein Parlamentsmandat innezuhaben).

Die Verfassung sieht in Art. 47 drei Ebenen der staatlichen Verwaltung vor: die nationale, die Gliedstaaten und die lokale Verwaltungsebene. Ausdrücklich wird ein dezentrales Regierungssystem (decentralized system of government) angestrebt und an zahlreichen Stellen in der Verfassung betont, lokale Kompetenzen sind jedoch nur relativ schwach ausgeprägt. Die föderalen Strukturen sind schwach ausgebildet, sodass allenfalls von einem ,gemäßigten Föderalismus" gesprochen werden kann. Die Vielzahl an Einschränkungen der Kompetenzen der Gliedstaaten sowie die schwache zweite Parlamentskammer ließen unter Umständen auch die Klassifizierung als dezentralen Einheitsstaat zu. Beispielsweise werden in der Verfassung zahlreiche Vorschriften zur Ausgestaltung von Gliedstaaten und lokaler Ebene gemacht und

10 Kernkriterium für die Unterteilung von präsidentiellen und parlamentarischen System ist bei Steffa$n i$ das Recht des Parlaments, die Regierung (d.h. insb. die Spitze der Regierung) aus politischen Gründen abzuberufen (Misstrauensvotum). Daneben stellt er weitere Kriterien für ein parlamentarisches System auf, zu denen die Abhängigkeit der Regierung von einer Parlamentsmehrheit sowie die generell enge Verbindung zwischen Parlamentsmehrheit und Regierung (z.B. in der Gesetzgebung), die Wahlfunktion des Parlaments, Fraktionsdisziplin und die politische Steuerungsaufgabe des Regierungschefs gehören. Zu einem präsidentiellen Regierungssystem gehören nach Steffani zum Teil das Parlamentsauflösungsrecht des Präsidenten, die Trennung von Ministeramt und Abgeordnetenmandat. Weiterhin ist die Exekutive in der Regel ,geschlossen“, d.h. aus nur einer Person bestehend (im Gegensatz zur „,doppelten“ Spitze aus Regierungschef und Staatsoberhaupt).Vgl. Winfried Steffani, Zur Unterscheidung parlamentarischer und präsidentieller Regierungssysteme, Opladen 1982, S. 392; Ders., Parlamentarische und präsidentielle Demokratie. Strukturelle Aspekte westlicher Demokratien, Opladen 1979.

11 Auch Lijphart bestimmt die parlamentarische Abrufbarkeit des „Head of Government“ als zentrales Kriterium für die Bestimmung eines parlamentarischen bzw. präsidentiellen Regierungssystems. Als zusätzliche Indikatoren führt er die Volkswahl der Exekutivspitze (direkt oder indirekt durch Wahlmänner/frauen) sowie die juristische Unterscheidung zwischen einer „Ein-Personen-Exekutive“ (alle Macht geht letztlich auf den Präsidenten zurück) im Gegensatz zur „kollegialen Exekutive“ an. Vgl. Arend Lijphart, Patterns of Democracy. Government Forms and Performance in Thirty-Six Countries, New Haven 1999. Zur Einteilung verschiedener Typen von pol. Systemen (8-Felder-Matrix) auch Arend Lijphart, Parliamentary versus Presidential Government, Reprint 2004, Oxford 1992, S. 6.

12 Nach Duverger besteht die Besonderheit des semi-präsidentiellen Systems in der Tatsache, dass der Staatspräsident vom Volk gewählt wurde, der Premierminister aber von einer Mehrheit im Parlament abhängig ist. Zum Semi-Präsidentialismus zählt er weiterhin, das Parlamentsauflösungsrecht des Präsidenten bzw. der Regierung, die Abberufbarkeit der Regierung, starke Kompetenzen des Präsidenten sowie die oben angesprochene „doppelte Exekutive“ aus Präsident und Premierminister. Vgl. Maurice Duverger, A New Political Model. Semi-Presidential Government, European Journal of Political Research 8 (1980), S. 166. 
kontrolliert. Die Justiz ist vollständig einheitsstaatlich organisiert. Den Gliedstaaten fehlt damit eine eigenständige Judikative.

Die derzeitige Verfassung von 2011 ist nach eigener Maßgabe eine Übergangsverfassung. Es wird ein „Permanenter Verfassungsprozess“ etabliert, welcher eine „endgültige“ Verfassung zum Ziel hat. Zu diesem Zweck wurde gemäß Art. 202 sechs Monate nach Inkrafttreten der Verfassung (2011) eine National Constitutional Review Commission eingerichtet. ${ }^{13} \mathrm{Im}$ Rahmen der Kommission sind eine Vielzahl an Konsultationsverfahren und -verpflichtungen festgelegt, die sowohl alle Parteien, als auch VertreterInnen der Zivilgesellschaft umfasst. Diese Kommission hat den Auftrag einen Verfassungsentwurf auszuarbeiten und dem Präsidenten vorzulegen (Art. 202 (10)). Dieser soll nach Beratungen eine „Nationale Verfassungsgebende Versammlung“ (National Constitutional Conference) einberufen (Art. 202 (11)), zu der die Verfassung umfangreiche Vorgaben macht und welche alle Teile der Gesellschaft beteiligen soll (Art. 203).

Auch die Änderung der bestehenden Verfassung ist mit dem (weltweit verbreiteten) Zustimmungsquorum von Zweidritteln beider Parlamentskammern möglich und an keinerlei weitere Vorgaben oder Einschränkungen gebunden (Art. 199).

\section{Parlament}

Der südsudanesische Parlamentarismus ist in Form eines asymmetrischen Bikameralismus ${ }^{14}$ mit zwei Parlamentskammern ausgestaltet, die gemeinsam die „National Legislature“ (Art. 54) bilden. Die erste Kammer ${ }^{15}$ (National Legislative Assembly) besteht aus auf nationaler Ebene direkt gewählten Abgeordneten (Art. 56) und vertritt die Interessen des Gesamtstaats. Beide Kammern werden für vier Jahre gewählt (Art. 66).

$\mathrm{Zu}$ den Kompetenzen der National Legislative Assembly gehören die klassischen Parlamentsrechte wie die Gesetzgebung, die Kontrolle der Regierung (inkl. Fragerechte und Impeachment-Möglichkeiten), das Budgetrecht sowie die Ratifikation internationaler Verträge (Art. 57). Artikel 68 regelt die Art und Länge der Sitzungswochen in beiden Kammern.

Die aktuelle Zusammensetzung der National Legislative Assembly basiert auf einer in der Verfassung (Art. 56 (2) bzw. Art. 94) bestimmten Interimslösung, wonach das Parlament aus 332 Sitzen besteht. Diese setzen sich aus den 170 Abgeordneten der Southern Sudan Legislative Assembly (SSLA) zusammen, die nach Maßgabe von Art. 57 (1) der Interim Constitu-

13 Vgl. Thomas Kenneth, South Sudan. President Assures Constitutional Review Commission of Unfettered Independence, 24.1.2012, http://allafrica.com/stories/201201251009.html (10.4.2013).

14 „Bikameralismus“ bezeichnet Parlamentssysteme mit zweier Kammern, der Begriff ,asymmetrisch“ soll auf das (auch im Vergleich zu anderen Staaten) enorme Kompetenzgefälle zwischen erster und zweiter Kammer hindeuten.

15 Zweite Kammern sind Parlamentskammern, „die nicht die allgemeine Volksvertretung darstellen, auch wenn sie im innerstaatlichen Sprachgebrauch auf Grund der historischen Entwicklung (Herrenhaus, Oberhaus) als „Erste Kammer“ bezeichnet werden“. Hans Albrecht Schwarz-Liebermann von Wahlendorf, Struktur und Funktion der sogenannten zweiten Kammer. Eine Studie zum Problem der Gewaltenteilung, Tübingen 1958, Abschnitt V. 
tion of the Southern Sudan 2005 gewählt wurden, den 96 südsudanesischen Abgeordneten des Parlaments der Republik Sudan (welche dort nach dem CPA Sitze erhielten) und 66 Abgeordneten, die der Präsident nach Art. 96 (2) lit. b bestimmt hat. Dieses Provisorium wird noch bis zur nächsten Parlamentswahl, also je nach Interpretation regulär bis 2015 anhalten.

Die zweite Kammer ${ }^{16}$ (Council of States) vertritt die Interessen der Gliedstaaten, deren Abgeordnete werden von den Parlamenten der Gliedstaaten gewählt (Art. 58). Die in Art. 59 festgelegten Rechte des Council of States sind nur untergeordneter Natur: mit einer sehr hohen Hürde (2/3 Mehrheit) können Regelungen zum dezentralen Regierungssystem vorgenommen werden und ansonsten die Regierung in Politikfeldern wie Reintegration, Kriegsfolgeschäden und Wiederaufbau kontrolliert werden.

Auch für den Council of States gibt es eine Interimslösung (Art. 58 (2) und 94 (3)), wonach es aus allen 20 SüdsudanesInnen, ${ }^{17}$ die während des CPA-Zeitraumes Mitglieder des Council of States der Republik Sudan waren, sowie 30 weitere vom Präsidenten ernannten MitgliederInnen besteht. „Zweite Kammern haben eine Repräsentationsfunktion und eine Kontrollfunktion [Kontrolle anderer Verfassungsorgane]“. ${ }^{18}$ Beide Funktionen werden vom Council of States nur unvollständig abgebildet, so versammeln sich zwar VertreterInnen der Gliedstaaten, jedoch sind die ihnen zugeteilten Gesetzgebungskompetenzen so schmal, dass sie nicht wirklich produktiv wirken können. Die Kontrolle der Regierung sowie Mitwirkung bei Ernennungen etc. obliegt der National Legislative Assembly. Der Council of States kann daher als schwache Zweite Kammer bezeichnet werden, ${ }^{19}$ was den Begriff des asymmetrischen Bikameralismus rechtfertigt.

Institutionalisierte OppositionsführerInnen in beiden Kammern (Minority Leader, Art. 71), stehen im Protokoll hinter Präsident, Vize-Präsident und den SprecherInnen der beiden Parlamentskammern. Dies deutet auf eine Anlehnung an das britische Parlamentssystem sowie den Versuch von power-sharing und Einbindung der Opposition durch eine funktionelle und gesamtstaatliche Funktionsvergabe hin.

Das Vorhandensein von parlamentarischen Ausschüssen in beiden Kammern (Art. 72) samt wissenschaftlichem Dienst (Art. 72 (4)) und die notwendige Beteiligung im Gesetzgebungsprozess (Art. $84(2)+(3)$ ) charakterisieren die südsudanesische Legislative als Arbeitsparlament bzw. einer Mischung aus Arbeits- und Redeparlament (analog etwa zum deutsche Bundestag), definitiv ist es kein reines Redeparlament.

16 Laut Haas haben ,zweite Kammern [...] in der Regel mindere Rechte und Kompetenzen innerhalb des politischen Prozesses [als die erste Kammer]“, vgl. Christoph M. Haas, Sein und nicht sein. Bikameralismus und die Funktion Zweiter Kammern, in: Gisela Riescher / Sabine Ruß / Christoph M. Haas (Hrsg.), Zweite Kammern, München 2010, S. 3.

17 Government of the Republic of South Soudan (GOSS), Council of States, 2012, www.goss-online. org/magnoliaPublic/en/states/Council-of-States.html (10.4.2013).

18 Haas, oben Fn. 16, S. 4. Haas unterteilt zweite Kammern in unitarisch, föderalisiert, föderal-unitarisiert, föderal und nach Direktwahl, indirekter Wahl, Legitimation, Nominierung, Ständevertretung, siehe S. 15.

19 Dazu Gisela Riescher / Sabine Ruß, Zur Funktion von Zweiten Kammern in modernen Demokratien, in: Gisela Riescher / Sabine Ruß / Christoph M. Haas (Hrsg.), Zweite Kammern, München 2010. 
Alle Abgeordneten (beider Kammern) müssen südsudanesische StaatsbürgerInnen sein und die in Art. 62 niedergelegten Voraussetzungen erfüllen, zu denen die Volljährigkeit (21 Jahre), der Vollbesitz geistiger Kräfte, die Fähigkeit des Lesens und Schreibens oder die Notwendigkeit, in den letzten sieben Jahre nicht rechtskräftig verurteilt worden zu sein, zählen. Umstände, unter denen Abgeordnete ihr Mandat verlieren sind in Art. 63 geregelt. Besonders auffällig ist Art. 63 Abs. 1 lit. f, nachdem Abgeordnete ihr Mandat verlieren, wenn sie zu einer anderen Partei wechseln. Hier scheint ein deutlich parteizentriertes Verständnis von MandatsträgerInnen durch. Einer strengen vertikalen Gewaltenteilung ist Art. 63 Abs. 1 lit. g geschuldet, wonach Abgeordnete ihr Mandat verlieren, sobald sie ein Regierungsamt auf einzelstaatlicher oder lokaler Ebene annehmen. Verliert ein Abgeordneter oder eine Abgeordnete ihr Mandat, finden Nachwahlen für diesen Sitz statt (Art. 64). Während ihrer Amtszeit genießen alle Abgeordneten Immunität (Art. $67+77$ ).

Eine systemische Besonderheit besteht in den weitreichenden Übergangsbestimmungen der Verfassung, die sowohl den südsudanesischen Abgeordneten des gesamtsudanesischen Parlaments, also auch die des gesamtsudanesischen Council of States (diese bestanden in der CPA-Periode 2005 bis 2009; geregelt in Art. 94) in das System integrieren und so zu Gunsten von power-sharing-Maßnahmen auf demokratische Neuwahlen verzichten.

\section{Staatspräsident, Regierung und Verwaltung}

Die Regierung besteht gemäß Art. 95 aus dem Präsidenten, dem Vize-Präsidenten (Art. 104+105) sowie den MinisterInnen und ihren StellvertreterInnen (Deputy Ministers, Art. 117).

Der Präsident wird für fünf Jahre (Art. 100(1)) direkt vom Volk gewählt (Art. 97(1)). Das Mindestalter beträgt 40 Jahre, alle weiteren Voraussetzungen entsprechen denen der Abgeordneten (Art. 98). Der Präsident ist Oberbefehlshaber der Streitkräfte (Art. 97(3)), gestaltet die Außenpolitik, schließt internationale Abkommen (Art. 101 lit. p) und vertritt den Südsudan nach innen und außen (Art. 101 lit. $\mathrm{m}+\mathrm{o}$ ). Er steht dem National Council of Ministers vor (Art. 101 lit. d). Weiter übt er klassische Rechte eines Staatsoberhaupt wie die Vergabe von Ehrungen (Art. 101 lit. 1), die Akkreditierung von BotschafterInnen (Art. 101 lit. o) sowie das Begnadigungsrecht (Art. 101 lit. h) aus. Der Präsident genießt während seiner Amtszeit Immunität vor Strafverfolgung und kann nur aufgrund schwerer Verstöße gegen die Verfassung mittels eines Impeachment-Verfahrens (Einleitung durch eine Zwei-Drittelmehrheit der $\mathrm{Na}$ tional Legislative Assembly und ein anschließendes Verfahren vor dem Obersten Gerichtshof (analog zu den USA)) sowie unter hohen Auflagen aus medizinischen Gründen vom Amt entfernt werden (Art. 103). Die sehr starke Stellung des Präsidenten zeigt sich insbesondere in der Übergangsperiode, wo er eine große Anzahl von Parlamentariern ernennt, aber auch darüber hinaus durch die Ernennung von RichterInnen und zahlreichen Kommissionsmit- 
gliederInnen. ${ }^{20}$ Diese starke Stellung birgt ein nicht unerhebliches Konfliktpotential für die Zukunft. ${ }^{21}$

Die Position des Vize-Präsidenten ist, zum Beispiel im Vergleich zu den Vereinigten Staaten von Amerika, schwach ausgestaltet. Der Vize-Präsident wird nicht vom Volk gewählt sondern vom Präsident berufen und von mit einer Zweidrittel-Mehrheit durch die National Legislative Assembly bestätigt (Art. 104 (1)), genauso erfolgt seine Absetzung (Art. 104(2)). Der Vize-Präsident vertritt den Präsidenten in seiner Abwesenheit und gehört dem National Council of Ministers sowie dem Security Council an (Art. 105).

Die Exekutive wird vom Kabinett (National Council of Ministers) geleitet. Es besteht aus dem Präsidenten, dem Vize-Präsidenten und den MinisterInnen (Art. 108 (2)) und ist die höchste exekutive Instanz des Südsudan (Art. 109(1)). Entscheidungen im Kabinett werden im Konsens oder mit einfacher Mehrheit getroffen (Art. 109 (2)) (Kabinettsprinzip; auch Art. 115 (3)). MinisterInnen werden durch den Präsidenten ernannt und von der National Legislative Assembly mit einfacher Mehrheit bestätigt (Art. $112(1)+(2)$ ), gleiches gilt für Deputy Minister (Art. 117). Der Präsident kann MinisterInnen nach eigenem Willen wieder entlassen (Art. 112 (1)). Die National Legislative Assembly kann mit einer Zweidrittel-Mehrheit der MitgliederInnen einem Minister oder einer Ministerin das Vertrauen entziehen, der Präsident muss diese(n) MinisterIn entlassen (Art. 118). Die Aufgaben des National Council of Ministers umfassen die aus anderen Staaten bekannten Funktionen (Art. 110). Kabinettsund MinisterInnenentscheidungen sind gerichtlich überprüfbar (Art. 116). Das Kabinett besteht aus der riesigen Zahl von 29 MinisterInnen und Ministerien, ${ }^{22}$ eine Zahl die bereits auf einen strukturell überdehnten Verwaltungsapparat, Kompetenzstreitigkeiten und Ineffizienzen, aber auch auf die Verteilung von Posten an potentielle Vetospieler hindeutet.

Für die Beschäftigten des Öffentlichen Dienstes (Art. 138) gibt die Verfassung besondere Regelungen und Prinzipien (Art. 139) vor. Zwei Institutionen, die Civil Service Commission (Art. 140) und Employees Justice Chamber (Art. 141) setzen sich für die Interessen und besonderen Belange des Öffentlichen Dienstes und seiner Beschäftigten ein.

\section{E. Gesetzgebung}

Das Recht formelle Gesetze (Gesetzgebungsakte) zu erlassen obliegt nach Art. 55 (1) bzw. Art. 164 (3) der Verfassung den Parlamenten (auf nationaler wie einzelstaatlicher Ebene), damit haben sie die Gesetzgebungskompetenz inne.

20 Siehe unten.

21 Zum System der Konfliktbearbeitung in der Verfassung des Südsudans und dessen strukturelle Probleme wird in einem mit diesem Artikel verwandten Beitrag ausführlicher eingegangen. Vgl. Ingo Henneberg / Friedrich Plank, Conflict resolution in the constitution of South Sudan 2011, in: Thilo Marauhn / Hatem Elliesie (Hrsg.), Legal Transformation in North Africa and the South Sudan, 2013, im Erscheinen.

22 Vgl. GOSS, Ministries, www.goss-online.org/magnoliaPublic/en/ministries.html (10.4.2013). Es gibt keine Minister ohne Geschäftsbereich bzw. Personen mit Kabinettsrang aber ohne Ministerium. 
$\mathrm{Zu}$ den Kompetenzen der nationalen Ebene gehören unter Anderem:

Außen-, Verteidigungspolitik, Staatsangehörigkeit, Passwesen, Nationale Symbole, Verkehr, Währung und Zentralbank, Postwesen, Wahl-, Partei- und Verfassungsfragen (Anhang A).

Das ordentliche Gesetzgebungsverfahren auf nationaler Ebene ähnelt stark dem gängigen Verfahren in anderen Staaten. Ein Gesetz durchläuft grundsätzlich drei Lesungen, bis es beschlossen und ausgefertigt wird (Art. 84 (1)).

Bei der ersten Lesung wird die Gesetzesvorlage durch einzelne Abgeordnete (beider Kammern), den Präsidenten, das Kabinett oder das Inter-House Committee eingebracht (Art. 83). Der Parlamentspräsident oder die Parlamentspräsidentin leitet den Entwurf danach dem zuständigen Parlamentsausschuss zur weiteren Beratung zu. Der Ausschuss berät die Vorlage, erstellt einen Bericht und schlägt gegeben falls Änderungen oder Ergänzungen vor. Weiterhin kann auch nach der zweiten Lesung ein Entwurf einem Ausschuss erneut zu Beratung vorgelegt werden (Art. 84 (2)). Sowohl ParlamentspräsidentIn (SprecherIn) als auch die bearbeitenden Ausschüsse haben die Möglichkeit externe Expertenmeinungen einzuholen und Hearings abzuhalten (Art. 84 (3)). Die obligatorische Anhörung von relevanten Interessensgruppen oder Akteuren der Zivilgesellschaft ist nicht vorgesehen.

In der zweiten Lesung wird der Gesetzesentwurf im Plenum debattiert und bewertet. Findet der Entwurf in der zweiten Lesung im Grundsatz eine Mehrheit, so gibt es eine dritte Lesung in der Details debattiert und Ergänzungen vorgenommen werden können. Danach wird der Entwurf in seiner finalen Version in einer letzten Lesung behandelt und als Ganzes oder abschnittsweise beschlossen (Art. 84 (2)). Hierzu ist in der Regel eine absolute Mehrheit notwendig (ordentliches Gesetzgebungsverfahren) (Art. 74 (1)).

Nachdem das Parlament ein Gesetz beschlossen hat wird es dem Präsidenten zur Unterzeichnung vorgelegt. Dieser hat danach 30 Tage Zeit um das Gesetz zu prüfen. Legt der Präsident in diesem Zeitraum kein Veto ein, so gilt das Gesetz als unterzeichnet und kann (vorbehaltlich einer im Gesetz bestimmten Frist) in Kraft treten (Art. 85 (1)). Legt er sein Veto ein, so wird das Gesetz und eine Begründung an das Parlament zurückverwiesen (Art. 85 (2)). Das Parlament hat daraufhin die Möglichkeit das Veto des Präsidenten durch eine ZweidrittelMehrheit in beiden Kammern zu überstimmen (Art. 85 (3)). Der Präsident hat danach keine weitere Veto-Möglichkeit und das Gesetz tritt in Kraft. Erreicht das Parlament diese sehr hohe Hürde nicht, so kann das Gesetz nicht in Kraft treten.

Neben dem ordentlichen Gesetzgebungsverfahren gibt es besondere Regelungen für die Haushaltsgesetzgebung. Zu Beginn des Haushaltsjahres bringt der Präsident einen Entwurf ein, der bestimmte in der Verfassung genannte inhaltliche Kriterien erfüllen muss (Art. 88 (1) lit. a-d). Das Parlament kann sie annehmen, ablehnen oder verändern (Art. 88 (3)), nach Zustimmung des Parlaments dürfen keinerlei finanzielle Änderungen am Haushaltsplan (Übertragung von Mitteln auf andere Haushaltsposten etc.) ohne Genehmigung des Parlaments vorgenommen werden (Art. 88 (6)).

Besondere Verfahrensvorschriften gibt es weiterhin für Gesetze, die Ressourcen und die Einkünfte aus diesen betreffen (Art. 87 (1)). Gesetze zu diesen Themen werden vom Präsi- 
denten zu Beginn des Haushaltsjahres eingebracht, das Parlament kann sie annehmen, ablehnen oder verändern (Art. $87(1)+(2))$.

Tagt das Parlament nicht (Sitzungsperiode siehe Abschnitt „Parlament“; Art. 68), so kann der Präsidenten „Provisorische Verordnungen“ (Provisional Orders) erlassen (Art. 86 (1)). Diese Verordnungen werden der zuständigen Parlamentskammer zugeleitet, sobald sie wieder tagt (Art. 86 (2)). Stimmt die Kammer der Verordnung zu, so erhält sie Gesetzeskraft, lehnt sie die Verordnung aber ab oder stimmt sie innerhalb ihrer Sitzungsperiode nicht zu, so erlischt die Rechtskraft der Verordnung (Art. 86 (3)). Ausgeschlossen ist der Erlass von Verordnungen, die gegen den eingangs erwähnten Verfassungskern verstoßen (Art. 86 (5)).

Beide Parlamentskammern haben die - gängige - Möglichkeit in ihrem Zuständigkeitsbereich der Exekutive das Recht zu übertragen, Ausführungsbestimmungen und Präzisierungen eines Gesetzes (Subsidiary Legislation) vorzunehmen (Art. 92). Diese Bestimmungen werden der jeweiligen Kammer zu Zustimmung und möglichen Änderung vorgelegt.

Die nationale Verfassung gibt den, ausdrücklich genannten, Parlamenten der Gliedstaaten keine expliziten Vorgaben für das Gesetzgebungsverfahren vor. Verfassungen der einzelnen Gliedstaaten sind momentan noch im parlamentarischen Verfahren bzw. noch nicht verfügbar.

$\mathrm{Zu}$ den Kompetenzen der einzelstaatlichen Ebene gehören unter Anderem:

Lokale Regierungsführung, Schulwesen, Familienplanung, Stadt- und Landplanung, kulturelle und religiöse Fragen (Anhang B).

Zur konkurrierenden Gesetzgebung (nationale und einzelstaatliche Ebene gemeinsam) gehören weite Politikbereiche wie: Ökonomische und soziale Entwicklung, Wissenschaft, Gesundheits- und Bankwesen, Medienpolitik, Umweltschutz, Genderfragen und Wiederaufbau (Anhang C). Widersprechen sich nationale und einzelstaatliche Gesetzgebung, so gilt der Vorrang des nationalen Rechts bis zur Lösung der Streitfrage (Anhang E).

Nicht erfasste, oder in der Zukunft entstehende Politikfelder (Residual Powers) sollen nach Natur der Sache und Sachzusammenhang entweder der nationalen oder der einzelstaatlichen Ebene geregelt werden. Diese unklare Regelung birgt Konfliktpotential, denn es gilt weder ein generelles Subsidiaritätsprinzip noch ein eindeutige Auffangregelung (Anhang D).

\section{F. Wahlsystem, Parteiensystem \& Interessensverbände}

Über das südsudanesische Wahlsystem/recht ${ }^{23}$ lässt sich im Frühjahr 2012 noch keine Aussage treffen. Das Parlament berät über ein Wahlgesetz, ${ }^{24}$ obwohl die Verfassung nur eine Frist von drei Monaten bis In-Krafttreten (also Okt./ Nov. 2011) eines Wahlgesetzes vorsah (Art. 197 (2)). Verfassungsrechtlich ist ein solches Gesetz (National elections law) in Art. 56

23 Zur Bedeutung von Wahlen generell und in Transformationsprozessen vgl. Dieter Nohlen, Wahlrecht und Parteisystem, Opladen 2009, S. 27-60.

24 Vgl. International Foundation for Electoral Systems (IFES), Building South Sudan's Electoral System, 23.2.2012, www.ifes.org/Content/Publications/Articles/2012/Building-South-Sudans-Electora 1-System.aspx (10.4.2013). 
(1) lit. b und Art. 58 (1) lit. b angesprochen und dem Gesetzgeber der Auftrag zur Ausarbeitung und Verabschiedung erteilt. Weiterhin ist eine unabhängige National elections commission vorgesehen, welche einen Monat nach Inkrafttreten des Wahlgesetzes etabliert werden soll (in Art. 197 genannt, Bezüge darauf in Art. 97 (1) und $102(2)+163$ (6)).

\section{Die SPLM}

Das Parteisystem befindet sich im Aufbau und ist geprägt von der alles dominierenden Sudan People's Liberation Movement (SPLM), welche als politischer Arm der Sudan People's Liberation Army (SPLA) die Unabhängigkeit des Landes hauptsächlich erstritt. ${ }^{25}$ Mit den Worten der International Crisis Group (ICG): ,the SPLM is the dominant political force in South Sudan, enjoying a near total monopoly of power in government “. ${ }^{26}$

Die SPLM verfügt über eine elaborierte „Verfassung“ (2008 auf dem 2. Parteitag verabschiedet) welche eine Parteistrukturen von der Nationalen Ebene über die Gliedstaaten bis hin zur einzelnen Dörfern sowie Gremien für bestimmte Fachthemen und eine innerparteiliche Demokratie vorsieht, ${ }^{27}$ befindet sich jedoch immer noch in einer Transformationsphase zwischen früherer Bewegung hin zu einer Partei. Die Dominanz der Partei führt zu einem Mitgliederzuwachs (Anreiz) und internen Ausdifferenzierung. ${ }^{28}$ Mit der SPLM-DC gab es bereits eine erste Abspaltung. Zu den Zielen der SPLM gehören laut Parteistatut nicht weiter spezifizierte Allgemeinplätze wie: Demokratie, Gleichbehandlung, Gewaltenteilung, Dezentralisierung, Wahrung der Menschenrecht sowie Umweltschutz. Parteichef ist Präsident Kiir. ${ }^{29}$ Die Webseite der SPLM lautet: http://www.splmtoday.com/.

Nach einer Umfrage des International Republican Institute (IRI) liegen die Zustimmungsraten zur SPLM bei bis zu 84 Prozent. ${ }^{30} 251$ von 332 der Parlamentssitze (in der ersten Kammer) werden von der SPLM besetzt. ${ }^{31}$ Unter diesen Voraussetzungen ist der Aufbau einer funktionierenden parlamentarischen Opposition und die Möglichkeit eine demokratische Alternative zur Regierung zu etablieren nicht sehr einfach. Umso notwendiger ist in einer solchen Situation der Aufbau einer starken Zivilgesellschaft, einer unabhängigen Justiz und einer freien Presse.

25 Die Selbstbeschreibung der SPLM-Geschichte vgl. SPLM Today, The Rise and Evolution of the SPLM, 2008, www.splmtoday.com/index.php/about/what-is-the-splm (10.4.2013).

26 ICG, oben Fn. 4, S. 12.

27 SPLM Today, The Constitution of the SPLM, 2008, www.splmtoday.com/index.php/about/constitu tion-of-the-splm (10.4.2013). Zu den einzelnen Parteiorganen vgl. auch SPLM Today, National Organs, 2008, www.splmtoday.com/index.php/structure/national-level (10.4.2013).

28 Vgl. ICG, oben Fn. 4, S. 13.

29 SPLM Today, oben Fn. 27, Constitution.

30 Vgl. International Republican Institute, Survey of South Sudan Public Opinion, 6.-27.9.2011, www.iri.org/sites/default/files/2011\%20December\%205\%20Survey\%20of\%20South\%20Sudan $\%$ 20Public\%20Opinion\%2C\%20September\%206-27\%2C\%202011.pdf (10.4.2013), S. 49.

31 Vgl. Central Intelligence Agency, CIA World Factbook 2012, 2012, https://www.cia.gov/library/pu blications/the-world-factbook/geos/od.html (10.4.2013). 


\section{Oppositionsparteien}

Die Quellenlage über die südsudanesische Parteienlandschaft ist, von der Regierungspartei SPLM abgesehen, eher schlecht. Das US-State Department listet neun Oppositionsparteien im Südsudan auf (Stand: 22.12.2011):

African National Congress, National Congress Party, South Sudan Democratic Forum, SPLM for Democratic Change, Sudan African National Union, United Democratic Front, United Democratic Salvation Front, United Democratic Salvation Front-Mainstream, und United Sudan African Party. ${ }^{32}$

In dieser Aufstellung fehlen allerdings sowohl die South Sudan Communist Party (SSCP) als auch das Sudan Liberal Youth Forum (SSLYF). Im Folgenden werden alle 11 südsudanesischen Oppositionsparteien kurz vorgestellt:

- Der African National Congress (ANC-Sudan) wurde im März 2006 gegründet und sieht sich als Partei aller Sudanesen, ob Sudan oder Südsudan. Zuvor war der ANC als SSF organisiert und wurde aus der sudanesischen Regierungspartei National Congress Party ausgeschlossen. ${ }^{33}$ Parteivorsitzender ist Lt. General George Kongor Arop. ${ }^{34}$ Am 12. September 2008 schlossen der ANC und die SPLM eine Allianz, ab diesem Zeitpunkt in allen politischen Fragen zusammenzuarbeiten. ${ }^{35}$

- Die im (Nord-) Sudan herrschende National Congress Party (NCP), 1981 gegründet, ${ }^{36}$ war bis zur Unabhängigkeit des Südsudans auch dort aktiv. Deren südsudanesische Lokalführung wechselte kurz vor der Unabhängigkeit zur SPLM über. ${ }^{37}$ Die NCP hat mehrere Sitze im Parlament inne.

- Das South Sudan Democratic Forum (SSDF) ${ }^{38}$ wurde 2001, als Dachorganisation von südsudanesischen Parteien und zivilgesellschaftlichen Organisationen, in London gegründet. Gründungsmitglieder waren: South Sudan Civic Forum, United Democratic Salvation Front (UDSF), SPLM und SPLA, Southern Sudan Referendum Party, Southern Sudan Consensus Building und Peace Action for Sudan and Africa (PAFSA). ${ }^{39}$ Die Organisation arbeitet zwar eng mit der SPLM zusammen, kritisierte sie jedoch auch häufig und sprach sich insbesondere 2007 und 2009 gegen die Abspaltung aus. Vorsitzender ist (im April

32 Vgl. US-State Department, oben Fn. 3.

33 Vgl. ANC-Sudan, Announcements, o.J., www.anc-sudan.com/announcements.html (10.4.2013).

34 Vgl. ANC-Sudan, Membership, o.J., www.anc-sudan.com/membership.html (10.4.2013).

35 Vgl. ANC-Sudan, Documents, o.J., www.anc-sudan.com/documents.html (10.4.2013).

36 Vgl. Gurtong Trust - Peace \& Media Project, National Congress Party, o.J., www.gurtong.net/Gov ernance/PoliticalParties/NationalCongressParty/tabid/409/Default.aspx (10.4.2013).

37 Vgl. Sudan Tribune, NCP South Sudan office closes down as officials join SPLM, 8.7.2011, www.sud antribune.com/NCP-South-Sudan-office-closes-down,39460 (10.4.2013).

38 Das Forum steht in keinem Zusammenhang zur gleich abgekürzten Southern Sudan Defence Force.

39 Sudan Vision Daily, Home News. South Sudan Democratic Forum, 8.5.2008, www.sudanvisiondai ly.com/modules.php?name $=$ News \&file $=$ article $\&$ sid $=12718(10.4 .2013)$. 
2009 bestätigt) Dr. Martin Elia Lomuro, welcher auch Agrar- und Parlamentsminister in der Übergangsregierung 2005-2011 war. ${ }^{40}$

- Die Sudan People's Liberation Movement for Democratic Change (SPLM-DC) ist eine Abspaltung der SPLM, die sich am September 2009 aus Kritik an der neuen Führung um Salva Kiir Mayardit (nach dem Tod des im Südsudan sehr verehrten John Garang), denen die SPLM-DC einen Verrat der Ideale der Bewegung und unzureichende und undemokratische Herangehensweise an aktuelle Probleme vorwirft, gründete. ${ }^{41} \mathrm{Zu}$ den Zielen der SPLM-DC zählen die Etablierung eines freien, gerechten, demokratischen und dezentralen Regierungssystems und ein Sozialvertrag zwischen allen Gruppen des (Gesamt-) Sudan, die Wahrung der Selbstbestimmung der Südsudanesen und die Realisierung einer freiwilligen Einheit des gesamten Sudan. ${ }^{42}$ Die Unabhängigkeit des Südsudan 2011 wird kritisch gesehen und ein weiterhin stark dezentral-föderale Struktur auch innerhalb des Südsudan eingefordert. ${ }^{43}$ Partei-Vorsitzender ist Dr. Lam Akol Ajawin. ${ }^{44}$

- Die United Democratic Front (UDF) wurde 2003 gegründet und sprach sich für die Unabhängigkeit des Südsudan aus und unterstützte das CPA. Sie kritisiert die SPLM und die südsudanesische Regierung. Parteivorsitzender ist Peter Abdelrahman Sule, der 1994 in der SPLA-United aktiv war und zwischenzeitlich Abgeordneter im gesamtsudanesischen Parlament war. 2008 unterzeichnete die UDF ein Konsultationsabkommen zwischen der SPLM und den südsudanesischen Oppositionsparteien. ${ }^{45}$

- Die United Democratic Salvation Front (UDSF) wurde von südsudanesischen Rebellen 1997 gegründet. Sie setzte sich für die Selbstbestimmung des Südsudan sowie Demokratisierung ein. Mit Abschluss des CPA wurde die UDSF Mitglied der nationalen Einheitsregierung sowie der südsudanesischen Übergangsregierung. Der Parteivorsitzende Dr. Joseph Malual Dong, der auch mehrfach Minister war, ${ }^{46}$ wurde im September 2011 das Misstrauen ausgesprochen und sein Amt bis zur Neuwahl mit Martin Tako Moyi besetzt.

40 Vgl. Sudan Electionnaire, South Sudan Democratic Forum, o.J., http://sudan.electionnaire.com/ind ex2.php (10.4.2013). Sudan Electionnaire ist eine Kooperation der Friedrich-Ebert-Stiftung und der Universität Khartum. Eine eigene Internetseite hat das SSDF nicht.

41 SPLM-DC, The Highlights of the SPLM-DC Founding National Delegates' Congress and Future Prospects, 14.9.2009, www.splm-dc.org/highlights-splm-dc-founding-national-delegates $\% \mathrm{E} 2 \% 80$ \%99-congress-and-future-prospects (10.4.2013).

42 SPLM-DC, oben Fn. 41.

43 Vgl. SPLM-DC, SPLM-DC Statement on the Independence of South Sudan, 7.07.2011, www.splm -dc.org/splm-dc-statement-independence-south-sudan (10.4.2013).

44 Vgl. SPLM-DC, oben Fn. 41.

45 Vgl. Sudan Electionnaire, United Democratic Front, o.J., http://sudan.electionnaire.com/index2. php (10.4.2013). Eine eigene Internetseite hat das UDF nicht.

46 Vgl. Sudan Electionnaire, United Democratic Salvation Front, o.J., http://sudan.electionnaire.com/i ndex2.php (10.4.2013). Eine eigene Internetseite hat das UDSF nicht. 
Dong wurde Verstöße gegen Parteistatuten und eine intransparente, tyrannische Führung vorgeworfen. ${ }^{47}$

- Die Sudan African National Union (SANU) (Ittihad Al Sudan Al Ghaoumi Al Afrighi) wurde 1962 als Sudan African Closed Districts National Union gegründet und 1963 nach Unterstützung eines UN-Plans für den „Südlichen Sudan“ in SANU umbenannt. SANU hat fünf Sitze im südsudanesischen Parlament. Ziele der Partei sind der Kampf gegen Tribalismus und Nepotismus, als Gefahr einer angestrebten ,unity in diversity“; sowie ein starker Föderalismus. Parteivorsitzender ist seit 1998 (zuletzt im April 2009 bestätigt) Dr. Toby Maduot Parek. ${ }^{48}$

- Über die United Democratic Salvation Front Mainstream (UDSF-M), vermutlich eine Abspaltung der UDSF, ist nur wenig bekannt. Zu ihren Zielen gehören wohl hauptsächlich die Verbesserung des Gesundheits- und Bildungssystems des Südsudan. Parteivorsitzender war im Sommer 2010 Gabriel Changson Chang. ${ }^{49}$

- Die United Sudan African Party (USAP) wurde 1987 als Dachorganisation von sieben südsudanesischen Parteien gegründet und ist der SPLM eng verbunden, beriet während der CPA-Verhandlungen aber auch die NCP. Nach dem power-sharing Absprachen des CPA erhielt die USAP 10 Sitze im gesamtsudanesischen Parlament, Parteivorsitzender Joseph Ukel wurde Minister in der Einheitsregierung. Die USAP favorisierte eine Einheit des Gesamtsudan mit starkem Föderalismus statt einer Abspaltung des Südsudan. 2008 unterzeichnete die USAP ein Konsultationsabkommen zwischen der SPLM und den südsudanesischen Oppositionsparteien. ${ }^{50}$

- Das Sudan Liberal Youth Forum (SSLYF) ging im Dezember 2011 aus der im Oktober 2010 gegründet der South Sudan Liberal Party (SSLP) hervor. Gründe waren die geringen politischen Gestaltungsmöglichkeiten und die starke demografischen Bedeutung der Jugend, weshalb man sich zugunsten des eigenen Jugendverbands aufgelöste. Eine Wiedergründung der SSLP ist nicht vor 2015 geplant. ${ }^{51}$ Das SSLYF ist verhältnismäßig gut organisiert und betreibt eine relativ umfangreiche Webseite. ${ }^{52} \mathrm{Zu}$ den Zielen der SSLYF gehören: Die Etablierung von Menschenrechten, Rechtstaatlichkeit, demokratische Re-

47 Vgl. Sudan Tribune, S. Sudan's opposition UDSF dismisses leader, 22.9.2011, www.sudantribune. com/South-Sudan-s-opposition-UDSF,40210 (10.4.2013).

48 Vgl. Gurtong Trust - Peace \& Media Project, Sudan African National Union, o.J., www.gurtong.ne t/Governance/PoliticalParties/SudanAfricanNationalUnion/tabid/410/Default.aspx (10.4.2013). Eine eigene Internetseite hat SANU nicht.

49 Vgl. Sudanese Online, Message of condolence from United Democratic Salvation Front-Main Stream (UDSF-M) Party, 2010, http://sudaneseonline.org/cs/blogs/articles/archive/2010/08/17/message-ofcondolence-from-united-democratic-salvation-front-main-stream-udsf-m-party.aspx (10.4.2013).

50 Vgl. Sudan Electionnaire, United Sudan African Party, o.J., http://sudan.electionnaire.com/index2. php (10.4.2013). Eine eigene Internetseite hat das USAP nicht.

51 Vgl. Africa Liberal Network, South Sudan Liberal Youth Forum - South Sudan, o.J., www.africali beralnetwork.org/standard.aspx?i_PageID=13295 (10.4.2013).

52 Vgl. http://sslyf.org/. 
gierungsführung inkl. freier und fairer Wahlen, die Sicherstellung von Frauen-, Minderheiten- und religiösen Rechten, der Kampf gegen Korruption und die Etablierung einer freien Marktwirtschaft im Südsudan. ${ }^{53}$ Vorsitzender ist seit Dezember 2011 Simon Achien Majur. ${ }^{54}$

- Die South Sudan Communist Party (SSCP) wurde Juni 2011 als lokale Ausgründung der im Sudan aktiven Sudanese Communist Party (SCP, gegründet 1946). Sie setzt sich für einen demokratischen und säkularen Staat ein und kritisiert die SPLM-Regierung, insbesondere in Fragen des Parteiengesetzes, stark. ${ }^{55}$ Parteivorsitzender (Parteisekretär) ist General Joseph Wol Modesto. ${ }^{56}$

Im November 2011 schlossen sich Democratic Forum, United Democratic Salvation Front Mainstream, United Sudan African Party und der African National Congress im Gliedstaat Unity zu einem Vier-Parteibündnis zusammen um gemeinsam eine wirksamere Opposition gegen die dominierende SPLM bilden zu können, welche sich unkooperativ zeigte. ${ }^{57}$ Zusammenfassend können die Oppositionsparteien nur als politisch wie organisatorisch schwach eingeschätzt werden, ${ }^{58}$ sie können in der jetzigen Verfassung kaum wirksame politische Alternativen zur SPLM anbieten.

\section{Interessenverbände}

Interessenverbände im westlichen Verständnis sind im Südsudan noch kaum vorfindbar. ${ }^{59} \mathrm{Zu}$ ihnen könnten in erster Linie Wirtschaftsverbände, Gewerkschaften und Berufsverbände zählen.

Am ehesten haben noch die großen Firmen, besonders im Öl-Sektor, die Möglichkeit einzeln oder zusammengeschlossen auf die Politik Einfluss zu nehmen. Für diesen Sektor gibt es aufgrund seiner immensen Bedeutung für die Wirtschaft des Landes besondere administrative Regelungen (ein eigenes Öl-Ministerium) und sogar Regelungen in der Verfassung (Art. 173-176, 178). Interessant sind im Zuge der schwach ausgeprägten Staatlichkeit auch

53 Vgl. South Sudan Liberal Youth Forum (SSLYF), Introduction, o.J., http://sslyf.org/?page_id=12 (10.4.2013).

54 Vgl. SSLYF, SSLYF general meeting conducted, 18.12.2011, http://sslyf.org/?p=259 (10.4.2013).

55 Vgl. Kerryn Williams, South Sudan. Africa's newest communist party, 16.12.2011, Links - International Journal of Socialist Renewal, http://links.org.au/node/2655 (10.4.2013).

56 Vgl. Dhieu Williams, Sudan. Local Communists Call for Secular Democratic State, 11.11.2011, http://allafrica.com/stories/201111210837.html (10.4.2013).

57 Vgl. Sudan Tribune, Opposition parties form coalition in Unity state, accuse SPLM of neglect, 1.12.2011, www.sudantribune.com/Opposition-parties-form-coalition,40872 (10.4.2013).

58 Vgl. ICG, oben Fn. 4, S. 16.

59 Zur Verbändetheorie vgl. Josef Schmid, Verbände. Interessenvermittlung und Interessenorganisation, München 1998, S. 64 f. 
CSR-Aktivitäten in diesem Wirtschaftssektor. ${ }^{60}$ Wie oben angesprochen gibt es Regelungen zur Organisation der Beschäftigten des Öffentlichen Dienstes. Gruppierungen und Zusammenschlüsse im zivilgesellschaftlichen Bereich werden im nächsten Abschnitt angesprochen.

\section{G. Politische Kultur, Partizipation, Zivilgesellschaft und unabhängige Institutionen}

Wie bereits in der Einführung angedeutet ist politische Kultur eine abstrakte Materie, die einem stetigen Wandel unterworfen ist. Politische Kultur ist daher grundsätzlich, auch in etablierten Demokratien, nur schwer messbar. ${ }^{61}$

In jedem Fall ist politische Kultur nicht einfach „da“, sondern muss sich mit dem verknüpften politischen System erst entwickeln. Dazu braucht es feste Institutionen, Arenen der politischen Debatte und vor allem stetige politische Praxis. Aus diesem Grund ist es im Frühjahr 2012 grundsätzlich noch zu früh, um von einer politischen Kultur des Südsudan zu sprechen. Der Staat ist gerade einmal etwas mehr als ein halbes Jahr alt und Institutionen wie das Parlament (obwohl es in der Transitionsphase schon bestand) benötigen mehr Kontinuität und Praxis um eine politische Kultur zu entwickeln. Politische Kultur ist allerdings nicht auf die formellen Institutionen beschränkt, sondern schließt Diskussionsprozesse in den Medien, Wahlkämpfe und Engagements der BürgerInnen mit ein.

Zum jetzigen Zeitpunkt liegt es bei der starken Dominanz der SPLM in Regierung und Parlament nahe, von einer sehr zentralisierten, teilweise gar autoritären politischen Kultur zu sprechen - ob sich dies nachhaltig ändert ist dem Verhalten aller relevanten Akteure so wie insbesondere den nächsten Wahlen (dem Umgang mit ihnen, Wahlkampf, Anerkennung des Ergebnisses, ggf. geringere Bedeutung der SPLM und der Umgang damit) geschuldet.

Die Einbindung relevanter zivilgesellschaftlicher Akteure erfolgt neben den üblichen demokratischen Verfahren wie Wahlen und Abstimmungen (Referenden, Art. 195, welche allerdings nicht von den BürgerInnen selbst initiiert werden können) durch eine Vielzahl von meist unabhängigen Kommissionen, die für bestimmte für den Südsudan prägende Politikfelder zuständig sind. Zu nennen sind hierbei die in der Verfassung besonders stark institutionalisierte Human Rights Commission (Art. 145 + 146), sowie die ebenfalls in der Verfassung vorgesehenen Anti-Corruption Commission (Art. 143), ${ }^{62}$ den Public Grievances Chamber (eine Art nationale Beschwerdestelle, Art. 147), die Relief and Rehabilitation Commissi-

60 CSR: corporate social responsibility. Eine interessante Studie zu CSR im Südsudan vgl. Kate Ives / Marc Buchner, Implementing CSR in South Sudan. Challenges faced by the oil sector in implementing international human rights, 2011, www.gurtong.net/LinkClick.aspx?fileticket=Q6NA7RfIBPk $\% 3 \mathrm{~d} \&$ tabid=124 (10.4.2013).

61 Dazu Gabriel A. Almond / Sidney Verba, Civic Culture. Political Attitudes and Democracy in Five Nations, Princeton 1963

62 Wobei die Anti-Corruption Commission bisher als wenig erfolgreich eingeschätzt werden kann. Vg1. Horand Knaup, Verschwundene Ölmilliarden. Gierige Elite treibt Südsudan in den Staatsbankrott, 10.6.2012, www.spiegel.de/politik/ausland/der-suedsudan-steht-ein-jahr-nach-der-unabhaengigkeit -vor-dem-bankrott-a-837370.html (10.4.2013). 
on (Art. 148), die Demobilization, Disarmament and Re-Integration Commission ${ }^{63}$ (Art. 149) und schließlich die HIV/AIDS Commission (Art. 150). Daneben gibt es eine größere Anzahl weiterer Kommissionen und Komitees für unterschiedlichste Politikbereiche, die allerdings nicht durch die Verfassung vorgesehen sondern per Gesetz oder Präsidialerlass gegründet wurden. ${ }^{64}$

Weitere wichtige unabhängige staatliche Institutionen sind die Bank of South Sudan (Art. 182), das National Audit Chamber (Art. 186) sowie das National Bureau of Statistics ${ }^{65}$ (Art. 193).

\section{H. Massenmedien}

Die Presse- und Medienlandschaft des Südsudan befindet sich noch im Aufbau. Es fehlt an ausgebildeten JournalistInnen, notwendiger Technik und teils auch an Papier für Druckerzeugnisse. Da die Infrastruktur des Landes sehr schlecht ist, kann eine flächendeckende Berichterstattung und damit die Lenkung der Aufmerksamkeit auf Probleme in ländlichen Gebieten kaum gewährleistet werden. Der Großteil der vorhandenen Medien ist in der neuen Hauptstadt Juba konzentriert. Die Verfassung garantiert zwar die Presse- und Meinungsfreiheit (Art. 24), in der Praxis müssen sich Eliten in Regierung, Verwaltung und Wirtschaft aber erst noch an stetige Kritik durch die Medien (Stichwort: Vierte Gewalt) gewöhnen. Ende 2011 kam es zu Verhaftungen von Journalisten und staatlichem Vorgehen gegen freie Presse, namentlich gegen die beiden Zeitungen The Destiny und Citizen. ${ }^{66}$ Für die Regulierung der Presse ist die nationale Ebene zuständig (Anhang A - Ziffer 45).

Bereits seit 2004 besteht mit der Union of Journalists of South Sudan ${ }^{67}$ eine Organisation, die für die Pressefreiheit und die Rechte von JournalistInnen eintritt. Sie ist Hauptansprechpartner der Regierung in Medienfragen. Die Medienlandschaft ist noch dünn, ausländische Geber wie die EU unterstützen Medienprojekte zur Stärkung der Zivilgesellschaft.

Eine kurze Liste südsudanesischer Medien (Stand: Mitte 2012, ohne Anspruch auf Vollständigkeit):

- Bhagita Radio (katholisch-religiös) (http://www.bakhitaradio.org/)

- Juba Post (http://jubapost.org/, derzeit nicht funktionierend)

63 Vgl. Republic of South Sudan DDR Commission, Home, 2012, www.ssddrc.org/ (10.4.2013).

64 Vgl. GOSS, Independent Commissions and Chambers, 2012, www.goss-online.org/magnoliaPublic /en/Independant-Commissions-and-Chambers.html (10.4.2013).

65 Weitere Informationen unter: http://ssnbs.org/ (10.4.2013).

66 Vgl. Benno Müchler, Presse im Südsudan. Diesel für die News-Hütte, 20.12.2011, www.sueddeuts che.de/medien/presse-im-sudan-diesel-fuer-die-news-huette-1.1239263 (10.4.2013).

67 Vgl. Media Diversity Institute, UJOSS representative met with South Sudan Minister of Information, 7.4.2011, www.media-diversity.org/mdi/index.php?option=com_content\&view $=$ article \&id=1808:u joss-representative-met-with-south-sudan-minister-of-information\&catid=136:mdis-work-in-south -sudan\&Itemid=209 (10.4.2013) und Publish What You Pay Norway, Union of Journalists of South Sudan (UJOSS), 2009, www.publish-whatyoupay.no/union-journalists-south-sudan-ujoss (10.4.2013). 
- Miraya FM(Radio) (United Nations Mission in Sudan; Geldgeber: Deutschland, Schweiz) (www.mirayafm.org)

- South Sudan 24 (eine News-Sammelseite verschiedener Quellen) (http://www.southsuda $\mathrm{n} 24 . \mathrm{com} /)$

- South Sudan Nation (Betreiber unklar) (http://southsudannation.com/)

- South Sudan News Agency (Sitz in den USA) (http://www.southsudannewsagency.com/)

- The Citizen (http://thecitizenews.com/, Webseite z.Z. abgeschaltet)

- The Destiny (keine Webseite)

- The People's Voice ${ }^{68}$ (Kooperation Union of Journalists of South Sudan und Media Diversity Institute (London); Geldgeber: EU)

- The Southern Eye Magazine (Juba, keine Webseite)

- Upper Nile Times (http://www.untimes.org/)

\section{Rechtssystem}

Art. 122 spricht die Rechtsprechung einer unabhängigen Judikative zu (auch: Art. 124), welche an Verfassung und Gesetze gebunden ist (Art. 122 (4)).

Das südsudanesische Justizsystem ist zentralstaatlich organisiert, es gibt einen nationalen Instanzenzug und keine eigene Gerichtsbarkeit in den Gliedstaaten (Art. 122-134 iVm. Anhang A Nr. 8). Es gilt ein einheitlicher Instanzenzug, ohne funktionale Aufteilung in Zivil-, Straf- oder Verwaltungsgerichte. Zentrale Stellung nimmt die Position des Chief Justice ein, welche der gesamten Judikative administrativ vorsteht (Art. 122 (8) und Art. 127) und wie sein Stellvertreter, der Deputy Chief Justice (Art. 128), Teil des Obersten Gerichtshofs ist.

Der Oberster Gerichtshof (Supreme Court) ist das höchste Gericht des Südsudan (Art. 125+126). Neben dem/ der angesprochenen Chief Justice und dem/ der Deputy Chief Justice setzt es sich wenigstens neun weiteren RichterInnen zusammen (Art. 125+126). Der Oberste Gerichtshof fungiert als Verfassungsgericht und Hüter der nationalen wie Gliedstaaten- Verfassungen (Art. 126 (1)), ist als oberstes Gericht im Instanzenzug für nationales wie Gliedstaaten-Recht zuständig (Art. 126 (2) lit. b), entscheidet Streit zwischen Staatsorganen (Art. 126 (2) lit. c+j), ist oberste Berufungs- und Revisionsinstanz in zivil-, straf- und verwaltungsrechtlichen Belangen (Art. 126 (2) lit. e+i), kann Gesetze aufgrund von Verstößen gegen die Verfassung aufheben und für nichtig erklären (Art. 126 (2) lit. d) und richtet über oberste RepräsentantInnen des Staates (Art. 126 (2) lit. f+g). Der Oberster Gerichtshof entscheidet in der Regel in Kammern zu jeweils drei RichterInnen, bei Verfassungsfragen im Plenum (Art. 126 (3)). Entscheidungen des Obersten Gerichtshofs sind endgültig und bindend (Art. $126(5))$.

Für die ordentliche Gerichtsbarkeit in allen Streitsachen, außer solchen, in den ausdrücklich direkt der Oberste Gerichtshof zuständig ist, sind zunächst County Courts (Art. 131),

68 Vgl. John Actually, Civilians Continue to Suffer at Hands of Rebels in Jonglei, 2011, www.mediadiversity.org $/ \mathrm{mdi} /$ index.php?option $=$ com_content\&view=section\&layout=blog\&id=31\&Itemid= 208 (10.4.2013). 
darüber sind als Appellationsinstanz die High Courts (Art. 130) und über diesen die Courts of Appeal (Art. 129) zuständig. Einzelheiten wie Errichtung, Zusammensetzung, Kompetenzen, Prozeduren und mögliche Rechtsbefehle der einzelnen Gerichte werden durch die Verfassung nicht geregelt und stehen jeweils unter Gesetzesvorbehalt. Alle RichterInnen werden vom Präsidenten auf Vorschlag einer unabhängigen National Judicial Service Commission (Art. 132) ernannt (Art. 133 (1)+(2)) Die Ernennung der Richter am Obersten Gerichtshof (inkl. Chief Justice und Deputy Chief Justice) und benötigt darüber hinaus die Zustimmung einer Zweidrittel-Mehrheit der National Legislative Assembly (Art. 133 (3)). Die National Judicial Service Commission ist auch für Disziplinarmaßnahmen gegen RichterInnen zuständig (Art. 134). Die Stellung von StaatsanwältInnen und AnwältInnen wird staatlich geregelt (Art. 135). Des Weiteren wird eine unabhängige Law Review Commission etabliert, dessen genaue Funktion in der Verfassung allerdings unklar bleibt (Art. 137).

Die Polizei ist, genauso wie der Strafvollzug (Art. 156 (3)), sowohl auf nationaler als auch auf Ebene der Gliedstaaten organisiert (Art. 155 (3)) und an Recht und Gesetz sowie insbesondere die Verfassung die darin enthaltenen Grundrechte gebunden (Art. 155 (6)). Sie setzt die Anordnungen der Judikative um (Art. 155 (6)).

\section{J. Regionalpolitik}

Die nationale Verfassung gibt allen zehn Einzelstaaten ${ }^{69}$ einen Rahmen vor, wie das einzelstaatliche politische System auszugestalten ist. So werden ein Länderparlament (State Legislative Assembly) in Art. 164 und die Exekutive in Form eines Gouverneurs oder einer Gouverneurin (Art. 165) und StellvertreterInnen sowie MinisterInnen festgeschrieben (Art. 165). Ungewöhnlich ist die in Art. $101 \mathrm{r}$ geregelte Möglichkeit, GouverneurInnen im Falle einer Krise im Einzelstaat welche die nationale Sicherheit bedrohen könnte, durch den Präsidenten abzuberufen werden kann. Der Ausgestaltung und Organisation der lokale Regierungs- und Verwaltungsebene durch die einzelnen Gliedstaaten werden in Art. 163 gewisse Vorgaben gemacht, unter Anderem, dass der lokalen Verwaltung ausreichend Mittel zur Verfügung gestellt werden müssen und lokale Amtsträger nach demokratischen Grundsätzen gewählt werden müssen. Wesentlicher sind Aufgabenzuweisungen in Art. 166 (6) und die Vorgabe einer dreigliedrigen Verwaltungsstruktur (Art. 166 (5)), welche die ländlichen Regionen in Counties (Bezirke), Payams und Bomas sowie urbane Gebiete analog in Cities, Municipals und Towns unterteilt. Die gesamte Regionalpolitik der Gliedstaaten steht nach Art. 166 (3) unter der Kontrolle eines durch den Präsidenten eingesetzten Local Government Board.

Am Beispiel des Western Equatoria State lässt sich die lokale Verwaltungsstruktur in Ansätzen nachvollziehen: Counties werden dort von ernannten CommissionerInnen sowie Executive DirectorInnen als ChefInnen der zivilen Verwaltung geleitet. Jeder County unterteilt sich in Payams und diese wiederrum weiter in Bomas. Payams und Bomas werden jeweils 
von einem Verwalter oder einer Verwalterin geführt. ${ }^{70}$ Durch die häufige Abgeschiedenheit ist die Kontrolle der lokalen Ebene schwierig,

„In practice, many commissioners operate like feudal lords, with few if any checks on their authority and their rule subject only to their personal relationship with the governor." ${ }^{\text {"71 }}$

Insgesamt sind, trotz des starken Postulats eines dezentralen Regierungssystems, dezentrale oder gar föderale Elemente in der Verfassung nur sehr schwach ausgeprägt und durch eine Vielzahl an Bestimmungen eingeschränkt. Der Nationalstaat dominiert eindeutig das staatliche Gefüge und lässt den Gliedstaaten nur wenige und relative unwichtige Regelungskompetenzen. Die zahlreichen Vorgaben durch die nationale Verfassung, die geringen Kompetenzen und insbesondere die schwache zweite Parlamentskammer, die Absetzbarkeit der Gouverneure sowie die Kontrolle des Local Government Board lassen an einer ernsthaften Dezentralisierungspolitik zweifeln. Die Charakterisierung des Südsudans als Bundesrepublik $^{72}$ muss unter diesen Voraussetzungen verworfen werden. Die zehn Staaten unterteilen sich in 86 Bezirke.

\section{K. Internationale Beziehungen}

Die internationalen Beziehungen des Südsudan sind geprägt vom angespannten Verhältnis zum Sudan. 20 Jahre Bürgerkrieg und Unterdrückung durch den Norden haben ihre Spuren hinterlassen. Ein Großteil der AmtsträgerInnen auf beiden Seiten wurde während des Konflikts sozialisiert. Grenzstreitigkeiten (besonders um die ölreiche Abyei-Region) und grenzüberschreitende Konflikte halten an und es kam zu neuerlicher militärischer Gewalt auf beiden Seiten. Eine zentrale Frage in den Beziehungen beider Staaten ist die zwingend erforderliche $^{73}$ Kooperation im Bereich der Erdölproduktion. Ein Großteil der vormals gesamtsudanesischen Erdölfelder liegt nun im Südsudan, welcher aber aufgrund des fehlenden Meerzugangs kurz- und mittelfristig auf die vorhandene Pipelineinfrastruktur des Sudan zurückgreifen muss - hier gab es im August 2012 eine erste erfolgreiche Vereinbarung (10\$ pro durchgeleitetes Barrel Rohöl) ${ }^{74}$ von der abzuwarten bleibt, ob sie Bestand haben wird.

70 Vgl. Government of Western Equatoria State, About Western Equatoria - Political and Administrative Structure, 22.5.2011, www.westate.org/index.php/about-us/index.php?option=com_content\&v iew $=$ article $\&$ id $=11 \&$ Itemid $=44 \&$ limitstart $=2(10.4 .2013)$.

71 ICG, oben Fn. 4, S. 19.

72 Arte TV - Mit offenen Karten, Südsudan - ein neuer Staat in Afrika (1/2), April 2012, http://ddc.ar te.tv/folge/suedsudan-ein-neuer-staat-in-afrika-1-2 (28.4.2012), Jean-Christophe Victor an der Stelle 2 min, 13 sek.

73 Der südsudanesische Staatshaushalt hängt zu 93\% an den Erdöleinnahmen, der des Sudan zu 58\%. Vgl. European Coalition on Oil in Sudan (ECOS), Sudan's oil industry after the referendum. Conference Report, December 2011, www.ecosonline.org/reports/2011/\%5Eindex.html/Oil_conference report_Dec2010.pdf.html (10.4.2013), S. 8.

74 Vgl. Jeffrey Gettleman, Two Sudans Reach Deal on Fees for Oil Pipelines, 4.8.2012, www.nytimes .com/2012/08/05/world/africa/two-sudans-reach-deal-on-fees-for-oil-pipelines.html (10.4.2013). 
Der Südsudan ist Mitglied der Vereinten Nationen (seit 14.7.2011) sowie der Afrikanischen Union (seit 27.7.2011). Der Südsudan unterhält Botschaften in den USA, der EU (für alle EU-Staaten, zusätzlich in Norwegen, Großbritannien), Kanada sowie den Nachbarstaaten Kongo, Kenia, Äthiopien, Uganda; daneben in Südafrika, Zimbabwe, Ägypten und Eritrea.

Ziele der südsudanesischen Außenpolitik sind laut Verfassung (Art. 43) unter Anderem: Internationale Zusammenarbeit, wirtschaftliche Integration in Afrika, Förderung der Menschenrechte, friedlicher Nachbarschaft und die Bekämpfung von organisierter Kriminalität und Terrorismus.

Im Zentrum der Außenpolitik stehen deutlich die Beziehungen zum Sudan, daneben wird aber auch versucht, Beziehungen zu anderen Staaten aufzubauen und Kooperationen zu etablieren. Hierbei liegt der Fokus zum einen auf den unmittelbaren Nachbarstaaten, besonders Kenia ist als Handelspartner (Meerzugang) interessant, sowie auf international wichtigen wirtschaftlichen Akteuren bzw. internationalen Geldgebern der Entwicklungszusammenarbeit wie den USA, die EU(-Staaten) und China.

VertreterInnen des Südsudans in den internationalen Beziehungen ist primär der Präsident (Art. 101 lit. o), in Abstufung der Vize-Präsident sowie die MinisterInnen. Der Ratifizierung internationaler Abkommen muss die National Legislative Assembly zustimmen (Art. 86 (7)).

Die 7.000 Mann starke United Nations Mission in the Republic of South Sudan (UNMISS $)^{75}$ wurde durch Sicherheitsrat-Resolution 1996 vom 8.07.2011 zunächst für ein Jahr ${ }^{76}$ eingerichtet und mit der Konsolidierung von Frieden und Sicherheit und Hilfe bei der Entwicklung beauftragt.

\section{Ausblick}

$\mathrm{Zu}$ den größten Herausforderungen des Südsudans zählt kurz- und mittelfristig der Aufbau einer funktionierenden staatlichen Verwaltung, welche im gesamten Land Wirkung entfalten kann, dies ist insbesondere auf lokaler Ebene wichtig. Es gilt das Gewaltmonopol des Staates zu gewährleisten, Milizen zu entwaffnen, Stammeskämpfe zu verhindern und allgemeine Sicherheit herzustellen. Daneben ist es wichtig die Wirtschaft und insbesondere die Infrastruktur aufzubauen und alle Teile des Landes zu erschließen und einer einheitlichen Staatsgewalt zu unterstellen. Hürden sind dabei die Reintegration von Kämpfern und Flüchtlingen, der geringe Bildungsgrad der Bevölkerung und die schlechte Gesundheitsversorgung. In der Außenpolitik bleiben die angespannten Beziehungen zum Sudan und der Grenzkonflikt um die Abyei-Region auf Sicht wichtigstes Thema. Aus wissenschaftlicher Perspektive gilt es besonders einen Fokus auf die weitere Implementierung der Verfassung, die Schaffung tatsächlich handlungsfähiger Institutionen und der Gewährung rechtlicher Freiheiten zu legen. Insbesondere dem

75 Vgl. UN Peacekeeping, UNMISS. United Nations Mission in the Republic of South Sudan, 2011, www.un.org/en/peacekeeping/missions/unmiss/index.shtml (10.4.2013) und auch UN, UNMISS. United Nations Mission In South Sudan, 2011, http://unmiss.unmissions.org/ (10.4.2013).

76 Vgl. UN S/RES/1996 (2011), 8.7.2011, www.un.org/ga/search/view_doc.asp?symbol=S/RES/1996 $\% 282011 \% 29$ (10.4.2013). 
sogenannten ,permanenten Verfassungsprozess“ sowie des tatsächlichen Auslaufens der Übergangsbestimmung 2015 sollte große Aufmerksamkeit gewidmet werden.

\section{Zusammenfassung}

Der Südsudan ist eine gemäßigt föderale Republik mit einem präsidentiellen Regierungssystem und (besonders in der Übergangsperiode) sehr starkem Präsidenten. Dieser steht der Exekutive vor, die sich weiterhin aus einem Vize-Präsidenten und dem Kabinett zusammensetzt. Die Legislative besteht aus zwei Kammern mit sehr starker erster, auf nationaler Ebene direkt gewählter, Kammer und schwacher Zweiter Kammer (Vertretung der Gliedstaaten durch von den Gliedstaatenparlamenten entsandten Abgeordneten) (asymmetrischer Bikameralismus). Die Gliedstaaten haben eingeschränkte Kompetenzen, die lokale Verwaltung ist, trotz verfassungsrechtlichem Bekenntnis zur dezentralen Regierungsführung, schwach. Die Judikative besteht aus einem einheitlichen, also nicht nach Rechtsmaterien unterschiedenen, national organisierten Instanzenzug mit einem Obersten Gerichtshof (gleichzeitig Verfassungsgericht) an der Spitze. Es bestehen zahlreiche Übergangsbestimmungen, welche hauptsächlich das Parlament betreffen. Hier wurde versucht, Abgeordnete des bestehenden südsudanesischen Parlaments (SSLA) sowie südsudanesische Abgeordnete aus dem gesamtsudanesischen Parlament einzubinden, was (auch durch der „Auffüllung“ des Parlaments durch den Präsidenten) auf Kosten von Gewaltenteilung und Demokratieprinzip geht. Beim verfassungsgemäßen Auslaufen der Übergangsbestimmungen 2015 sind Präsident und erste Kammer die beiden Machtzentren der Republik. Die Verfassung garantiert Grund- und Menschenrechte und schützt bestimmte Staatsstrukturprinzipien vor willfähriger Änderung durch Präsidialdekrete. Die Parteilandschaft ist schwach ausgeprägt und durch die Regierungspartei SPLM dominiert. Die Medienlandschaft befindet sich im Aufbau und ist nicht frei von staatlichen Eingriffen. Interessenverbände sind noch kaum vorhanden. Die kaum organisierte Zivilgesellschaft soll durch eine Vielzahl von Fachkommissionen eingebunden werden. In der Außenpolitik dominieren die schlechten Beziehungen zum Sudan und ungeklärte Territorialfragen. Die Verfassung von 2011 ist nach eigener Maßgabe eine Übergangsverfassung und soll in einem breiten, festgelegten Verfahren durch eine neue Verfassung ersetzt werden. 


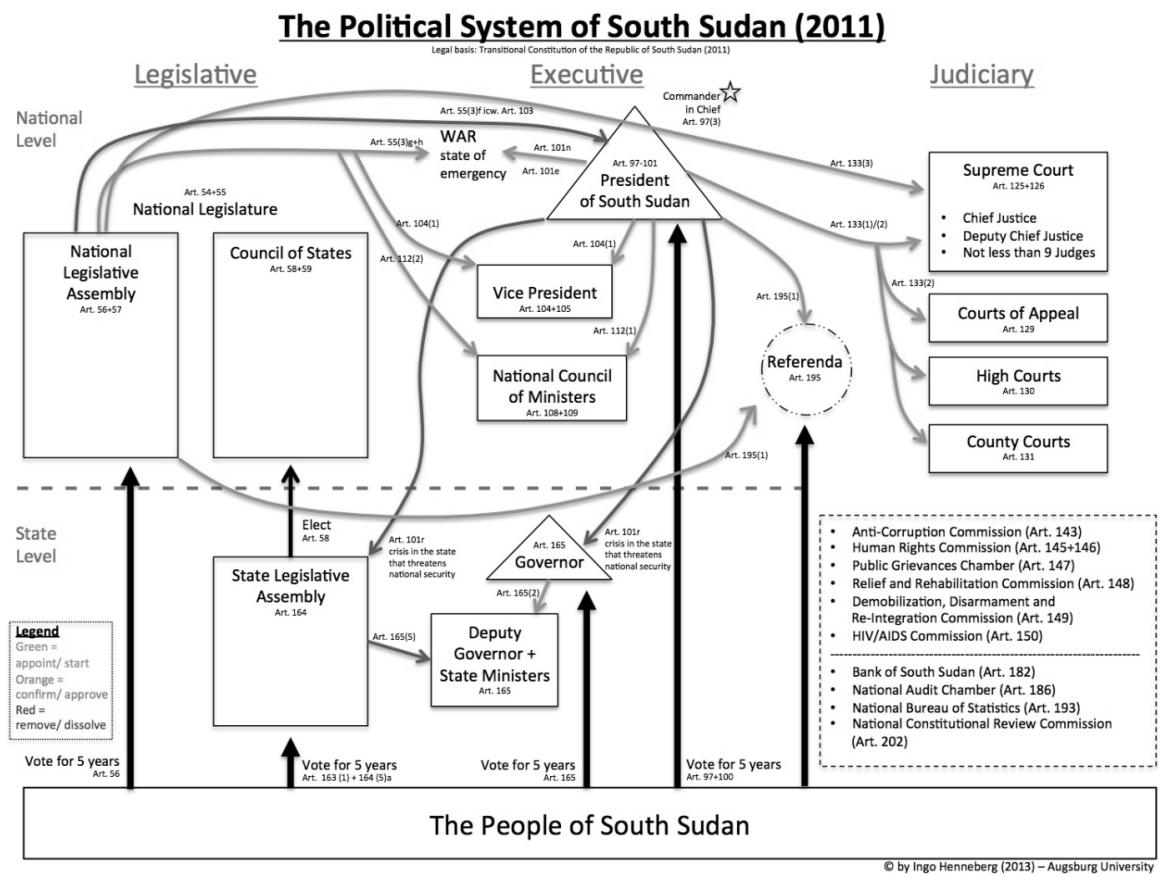

Abb. $1^{77}$

77 Eigene Darstellung. Dieses Diagramm zeigt grafisch die wichtigsten Institutionen des neuen südsudanesischen Staates und setzt sie in Beziehung zueinander. Aufgrund der Komplexität eines pol. Systems können nur die wesentlichen Staatsorgane auf nationaler Ebene sowie exemplarisch auf Ebene der Gliedstaaten, und deren Wahl durch das Volk dargestellt werden. Gleiches gilt für die Kompetenzen der einzelnen Institutionen, welche sich in der Darstellung hauptsächlich auf die Rechte der einzelnen Organe zur Nominierung, Bestätigung oder Absetzung eines jeweils anderen Staatsorgans konzentrieren. Daneben werden nur noch der Oberbefehl über die Streitkräfte, die Aktivierung des Kriegsfalls und Notstands sowie die Möglichkeit zu Initiierung von Referenden genannt. Auf die Darstellung der regionalen bzw. lokalen Regierungs- und Verwaltungsebene wurde (auch aufgrund der differenzierten Lage in verschiedenen Gliedstaaten) bewusst verzichtet.

Das Diagramm ist zunächst vertikal in die drei Gewalten Legislative, Exekutive und Judikative und horizontal in die nationale und die Gliedstaatenebene eingeteilt. Dicke schwarze Pfeile symbolisieren Wahlen und Abstimmungen durch das südsudanesische Volk, ein dünnerer schwarzer Pfeil die Wahl der Mitglieder des Council of States durch die jeweiligen Gliedstaaten-Parlamente. Grüne Pfeile bedeuten das Recht zu einer Position zu ernennen, ein Referendum zu starten oder den Krieg bzw. Notstand auszurufen. Orangene Pfeile bedeuten, das Recht einer Ernennung zuzustimmen und rote Pfeile das Recht eine Position abzuberufen, zu entlassen oder ein Impeachmentverfahren einleiten zu können. 